\title{
A CLASS OF TWISTED BRAIDED GROUPS*
}

\author{
Zhengming JiaO AND SHUANHONg WaNG
}

\section{Introduction}

As a dual concept of quasitriangular Hopf algebra, the coquasitriangular Hopf algebra was introduced by Larson and Towber [1] in 1991. In 1993, some properties of coquasitriangular Hopf algebra were studied by Doi [2]. In special, a class of coquasitriangular Hopf algebra construction $\left(A^{\sigma}, \tilde{\sigma}\right)$ was discovered. Recently Doi and Takeuchi [3] investigated a class of $A^{\sigma}$ type Hopf algebra $B \bowtie_{\tau}$ $H$ which is a special case of Majid's double crossproduct $B \bowtie H$ [4], [5]. So Doi's coquasitriangular Hopf algebra construction $\left(A^{\sigma}, \tilde{\sigma}\right)$ is an important significant coquasitriangular Hopf algebra. While Majid has introduced the braided group over coquasitriangular Hopf algebra in monoidal categories [6]. It is known that every coquasitriangular Hopf algebra $A$ can be converted by a process of transmutation into a braided group $\underline{A}$.

In this paper, we work with an invertible bilinear $\sigma$ on $A$ where $A$ is a Hopf algebra. In section 2, we introduce the concept of $T$-Hopf algebra where $T$ : $A \otimes A \rightarrow A \otimes A$ is a bilinear map. Then we show that $A_{\sigma}$ with the invertible 2-cocycle $\sigma$ [2], [3], [7] is a $T_{\sigma}$-Hopf algebra where $T_{\sigma}: A \otimes A \rightarrow A \otimes A$ is defined by

$$
a \otimes b \mapsto \sum \sigma\left(a_{1} S\left(a_{5}\right), b_{1} S\left(b_{5}\right)\right) b_{3} \otimes a_{3} \sigma^{-1}\left(b_{2} S\left(b_{4}\right), a_{2} S\left(a_{4}\right)\right)
$$

which is different from that of [2].

Next in section 3 , we give some necessary lemmas. In section 4 , we show that if $A$ is a commutative Hopf algebra, then $A_{\sigma}$ can be obtained as a braided group $\underline{A}^{\sigma}$. We work over a fixed field $k$ and follow Sweedler's book [10] for terminology on coalgebras, bialgebras and Hopf algebras. Let $C$ be a coalgebra, the sigma notation

$$
\Delta(c)=\sum c_{1} \otimes c_{2}
$$

for all $c \in C$ will be used frequently later. The antipode of a Hopf algebra will be denoted by $S$. We mainly refer to [2] and [3].

\footnotetext{
* This work was supported by the NSF of P.R. China and the Foundation of CSC.

Received March 16, 1999; revised July 7, 1999.
} 


\section{The $T_{\sigma}$-Hopf algebras $A_{\sigma}$}

In this section we will give the definition of the $T_{\sigma}$-Hopf algebra and construct a $T_{\sigma}$-Hopf algebra $A_{\sigma}$.

Let $A$ be a bialgebra and let $\sigma$ be an invertible bilinear form on $A$, here "invertible" means $\sigma$ has an inverse in the dual algebra $(A \otimes A)^{*}$. We say that $\sigma$ is a 2-cocycle if [2], [3], [7]:

$$
\sum \sigma\left(x_{1}, y_{1}\right) \sigma\left(x_{2} y_{2}, z\right)=\sum \sigma\left(y_{1}, z_{1}\right) \sigma\left(x, y_{2} z_{2}\right)
$$
1.6(a)]:

Let $\sigma^{-1}$ is the convolution inverse of $\sigma$, then we easily have [1, Theorem

$$
\sum \sigma^{-1}\left(x_{1} y_{1}, z\right) \sigma^{-1}\left(x_{2}, y_{2}\right)=\sum \sigma^{-1}\left(x, y_{1} z_{1}\right) \sigma^{-1}\left(y_{2}, z_{2}\right)
$$

We next define a coquasitriangular Hopf algebra [2] to be a pair $(A, \sigma)$, where $A$ is a Hopf algebra over $k$ and $\sigma$ is a bilinear form on $A$ satisfying the followings:

(BR1) $\sigma(x y, z)=\sum \sigma\left(x, z_{1}\right) \sigma\left(y, z_{2}\right)$;

(BR2) $\sigma(x, y z)=\sum \sigma\left(x_{1}, z\right) \sigma\left(x_{2}, y\right)$ :

(BR3) $\sigma(1, x)=\sigma(x, 1)=\varepsilon(x)$;

(BR4) $\sum \sigma\left(x_{1}, y_{1}\right) x_{2} y_{2}=\sum y_{1} x_{1} \sigma\left(x_{2}, y_{2}\right)$

for all $x, y, z \in A$.

The $\sigma$ satisfying condition (BR3) is called a normal bilinear form on $A$.

It is not hard to see that the following is true.

Lemma 2.1. Assume that $\sigma$ is an invertible 2-cocycle bilinear form on $A$, then we have the followings:

(1) $\sigma(x y, z)=\sum \sigma^{-1}\left(x_{1}, y_{1}\right) \sigma\left(y_{2}, z_{1}\right) \sigma\left(x_{2}, y_{3} z_{2}\right)$;

(2) $\sigma(x, y z)=\sum \sigma^{-1}\left(y_{1}, z_{1}\right) \sigma\left(x_{1}, y_{2}\right) \sigma\left(x_{2} y_{3}, z_{2}\right)$;

(3) $\sigma^{-1}(x y, z)=\sum \sigma^{-1}\left(x_{1}, y_{1} z_{1}\right) \sigma^{-1}\left(y_{2}, z_{2}\right) \sigma\left(x_{2}, y_{3}\right)$;

(4) $\sigma^{-1}(x, y z)=\sum \sigma^{-1}\left(x_{1} y_{1}, z_{1}\right) \sigma^{-1}\left(x_{2}, y_{2}\right) \sigma\left(y_{3}, z_{2}\right)$

for all $x, y, z \in A$.

Lemma 2.2 ([2, Theorem 1.6(b), (c)]). Let $A$ be a Hopf algebra with a normal invertible 2-cocycle $\sigma$, then:

(i) Define $A^{\sigma}=A$ as a coalgebra and

$$
\begin{gathered}
x \cdot y=\sum \sigma\left(x_{1}, y_{1}\right) x_{2} y_{2} \sigma^{-1}\left(x_{3}, y_{3}\right) ; \\
S^{\sigma}(x)=\sum \sigma\left(x_{1}, S\left(x_{2}\right)\right) S\left(x_{3}\right) \sigma^{-1}\left(S\left(x_{4}\right), x_{5}\right) .
\end{gathered}
$$

Then $A^{\sigma}$ is a Hopf algebra.

(ii) If $A$ is commutative as an algebra, then $\left(A^{\sigma}, \tilde{\sigma}\right)$ is a symmetric coquasitriangular Hopf algebra, where $\tilde{\sigma}$ is defined by

$$
\tilde{\sigma}(x, y)=\sum \sigma\left(y_{1}, x_{1}\right) \sigma^{-1}\left(x_{2}, y_{2}\right)
$$


DEFINITION 2.3. Let $\left(H, m_{H}, \Delta_{H}\right)$ be an algebra and a coalgebra (not necessary bialgebra). Let $T: H \otimes H \rightarrow H \otimes H$ be a linear map such that $(H \otimes H, \cdot T)$ is an algebra, where

$$
(a \otimes b) \cdot_{T}(c \otimes d)=\left(m_{H} \otimes m_{H}\right)(I \otimes T \otimes I)(a \otimes b \otimes c \otimes d) .
$$

$H$ is called a twist $T$-bialgebra if $\Delta_{H}$ is a algebra homomorphism. Furthermore, if there is an antipode for $H$, then $H$ is called a twist $T$-Hopf algebra.

EXAMPLE 2.4. Let $H$ be any Hopf algebra, and $\tau: H \otimes H \rightarrow H \otimes H$ is the classical twist, then $H$ is a twist $\tau$-Hopf algebra.

A left $H$-comodule coalgebra $C$ means $(C, \Delta, \varepsilon, \rho)$ where $(C, \Delta, \varepsilon)$ is a coalgebra and $\rho: C \rightarrow H \otimes C, \rho(c)=\sum c^{(1)} \otimes c^{(2)}$, for all $c \in C$ is the comodule structure map such that

i) $\sum c^{(1)} \varepsilon\left(c^{(2)}\right)=\varepsilon(c)$;

ii) $\sum c^{(1)} \otimes c^{(2)}{ }_{1} \otimes c^{(2)}{ }_{2}=\sum c_{1}^{(1)} c_{2}^{(1)} \otimes c_{1}^{(2)} \otimes c_{2}^{(2)}$.

In [9], Molnar has affirmed that $\left(H, \mathrm{Co}_{H}\right)$ forms a left $H$-comodule coalgebra $[9,(2.5)(\mathrm{a})]$, where $\mathrm{Co}_{H}$ is a coadjoint action on $H$ and is assigned to $\mathrm{Co}_{H}(h)=\sum h_{1} S\left(h_{3}\right) \otimes h_{2}$. Now we can obtain a class of twist $T$-Hopf algebra. $\sigma$ and

Proposition 2.5. Let $A$ be a Hopf algebra with a normal invertible 2-cocycle

$$
\sigma(x y z, m n t)=\sigma(y x z, n m t)=\sigma(x z y, m t n)
$$

for all $x, y, z, m, n, t \in A$, then there is a $T_{\sigma}$-bialgebra $A_{\sigma}$ defined by

$$
\begin{gathered}
\Delta_{\sigma}(a)=\sum \sigma^{-1}\left(a_{1} S\left(a_{3}\right), a_{4} S\left(a_{6}\right)\right)\left(a_{2} \otimes a_{5}\right) ; \\
a \cdot_{\sigma} b=\sum \sigma\left(a_{1} S\left(a_{3}\right), b_{1} S\left(b_{3}\right)\right)\left(a_{2} b_{2}\right) ; \\
T_{\sigma}: A \otimes A \rightarrow A \otimes A, \\
T_{\sigma}(a \otimes b)=\sum \sigma^{-1}\left(b_{2} S\left(b_{4}\right), a_{2} S\left(a_{4}\right)\right)\left(b_{3} \otimes a_{3}\right) \sigma\left(a_{1} S\left(a_{5}\right), b_{1} S\left(b_{5}\right)\right) .
\end{gathered}
$$

Furthermore, if $S^{2}=I$ then $A_{\sigma}$ is a $T_{\sigma}$-Hopf algebra, and it's antipode is $S$.

Proof. 1. Coassociative law.

$$
\begin{aligned}
(I \otimes & \left.\Delta_{\sigma}\right) \Delta_{\sigma}(a) \\
& =\sum \sigma^{-1}\left(a_{1} S\left(a_{3}\right), a_{4} S\left(a_{11}\right)\right) \sigma^{-1}\left(a_{5} S\left(a_{7}\right), a_{8} S\left(a_{10}\right)\right)\left(a_{2} \otimes a_{6} \otimes a_{9}\right) \\
& =\sum \sigma^{-1}\left(a_{1} S\left(a_{3}\right), a_{4} S\left(a_{8}\right) a_{9} S\left(a_{13}\right)\right) \sigma^{-1}\left(a_{5} S\left(a_{7}\right), a_{10} S\left(a_{12}\right)\right)\left(a_{2} \otimes a_{6} \otimes a_{11}\right) \\
& =\sum \sigma^{-1}\left(a_{2} S\left(a_{4}\right), a_{7} S\left(a_{9}\right)\right) \sigma^{-1}\left(a_{1} S\left(a_{5}\right) a_{6} S\left(a_{10}\right), a_{11} S\left(a_{13}\right)\right)
\end{aligned}
$$$$
\left(a_{3} \otimes a_{8} \otimes a_{12}\right)
$$ 


$$
\begin{aligned}
& =\sum \sigma^{-1}\left(a_{2} S\left(a_{4}\right), a_{5} S\left(a_{7}\right)\right) \sigma^{-1}\left(a_{1} S\left(a_{8}\right), a_{9} S\left(a_{11}\right)\right)\left(a_{3} \otimes a_{6} \otimes a_{10}\right) \\
& =\left(\Delta_{\sigma} \otimes I\right) \Delta_{\sigma}(a) .
\end{aligned}
$$

2. Associative law.

$$
\begin{aligned}
\left(a \cdot{ }_{\sigma} b\right) & \cdot{ }_{\sigma} c \\
& =\sum \sigma\left(a_{1} S\left(a_{5}\right), b_{1} S\left(b_{5}\right)\right) \sigma\left(a_{2} b_{2} S\left(a_{4} b_{4}\right), c_{1} S\left(c_{3}\right)\right)\left(a_{3} b_{3} c_{2}\right) \\
& =\sum \sigma\left(a_{1} S\left(a_{5}\right), b_{1} S\left(b_{5}\right)\right) \sigma\left(a_{2} S\left(a_{4}\right) b_{2} S\left(b_{4}\right), c_{1} S\left(c_{3}\right)\right)\left(a_{3} b_{3} c_{2}\right) \\
& =\sum \sigma\left(a_{1} S\left(a_{3}\right), b_{2} S\left(b_{4}\right) c_{2} S\left(c_{4}\right)\right) \sigma\left(b_{1} S\left(b_{5}\right), c_{1} S\left(c_{5}\right)\right)\left(a_{2} b_{3} c_{3}\right) \\
& =\sum \sigma\left(a_{1} S\left(a_{3}\right), b_{2} c_{2} S\left(b_{4} c_{4}\right)\right) \sigma\left(b_{1} S\left(b_{5}\right), c_{1} S\left(c_{5}\right)\right)\left(a_{2} b_{3} c_{3}\right) \\
& =a \cdot_{\sigma}\left(b \cdot{ }_{\sigma} c\right) .
\end{aligned}
$$

3. $\Delta_{\sigma}$ is an algebra homomorphism.

$$
\Delta_{\sigma}\left(a \cdot{ }_{\sigma} b\right)
$$

$$
\begin{aligned}
= & \sum \sigma\left(a_{1} S\left(a_{8}\right), b_{1} S\left(b_{8}\right)\right) \sigma^{-1}\left(a_{2} b_{2} S\left(a_{4} b_{4}\right), a_{5} b_{5} S\left(a_{7} b_{7}\right)\right)\left(a_{3} b_{3} \otimes a_{6} b_{6}\right) \\
= & \sum \sigma\left(a_{1} S\left(a_{5}\right) a_{6} S\left(a_{10}\right), b_{1} S\left(b_{5}\right) b_{6} S\left(b_{10}\right)\right) \\
& \sigma^{-1}\left(a_{2} S\left(a_{4}\right) b_{2} S\left(b_{4}\right), a_{7} S\left(a_{9}\right) b_{7} S\left(b_{9}\right)\right)\left(a_{3} b_{3} \otimes a_{8} b_{8}\right) \\
= & \sum \sigma^{-1}\left(\left(a_{1} S\left(a_{5}\right)\right)_{1},\left(a_{6} S\left(a_{10}\right)\right)_{1}\right) \sigma\left(\left(a_{6} S\left(a_{10}\right)\right)_{2},\left(b_{1} S\left(b_{5}\right) b_{6} S\left(b_{10}\right)\right)_{1}\right) \\
& \sigma\left(\left(a_{1} S\left(a_{5}\right)\right)_{2},\left(a_{6} S\left(a_{10}\right)\right)_{3}\left(b_{1} S\left(b_{5}\right) b_{6} S\left(b_{10}\right)\right)_{2}\right) \sigma\left(\left(a_{2} S\left(a_{4}\right)\right)_{2},\left(b_{2} S\left(b_{4}\right)\right)_{3}\right) \\
& \sigma^{-1}\left(\left(a_{2} S\left(a_{4}\right)\right)_{1},\left(b_{2} S\left(b_{4}\right)\right)_{1}\left(a_{7} S\left(a_{9}\right) b_{7} S\left(b_{9}\right)\right)_{1}\right) \\
& \sigma^{-1}\left(\left(b_{2} S\left(b_{4}\right)\right)_{2},\left(a_{7} S\left(a_{9}\right) b_{7} S\left(b_{9}\right)\right)_{2}\right)\left(a_{3} b_{3} \otimes a_{8} b_{8}\right) \\
= & \sum \sigma^{-1}\left(a_{1} S\left(a_{9}\right),\left(a_{10} S\left(a_{14}\right)\right)_{1}\right) \sigma\left(\left(a_{10} S\left(a_{14}\right)\right)_{2},\left(b_{1} S\left(b_{9}\right) b_{10} S\left(b_{14}\right)\right)_{1}\right) \\
& \sigma\left(a_{2} S\left(a_{8}\right),\left(b_{2} S\left(b_{8}\right)\right)\left(a_{10} S\left(a_{14}\right)\right)_{3}\left(b_{10} S\left(b_{14}\right)\right)_{2}\right) \sigma\left(a_{4} S\left(a_{6}\right),\left(b_{4} S\left(b_{6}\right)\right)_{2}\right) \\
& \sigma^{-1}\left(a_{3} S\left(a_{7}\right),\left(b_{3} S\left(b_{7}\right)\right)\left(a_{11} S\left(a_{13}\right)\right)_{1}\left(b_{11} S\left(b_{13}\right)\right)_{1}\right) \\
& \sigma^{-1}\left(\left(b_{4} S\left(b_{6}\right)\right)_{1},\left(a_{11} S\left(a_{13}\right)\right)_{2}\left(b_{11} S\left(b_{13}\right)\right)_{2}\right)\left(a_{5} b_{5} \otimes a_{12} b_{12}\right) \\
= & \sum \sigma^{-1}\left(a_{1} S\left(a_{9}\right), a_{10} S\left(a_{18}\right)\right) \sigma\left(a_{11} S\left(a_{17}\right), b_{1} S\left(b_{9} b_{10} S\left(b_{18}\right)\right)\right. \\
& \sigma\left(a_{2} S\left(a_{8}\right),\left(b_{2} S\left(b_{8}\right)\right)\left(a_{12} S\left(a_{16}\right)\right)\left(b_{11} S\left(b_{17}\right)\right)\right) \sigma\left(a_{4} S\left(a_{6}\right),\left(b_{4} S\left(b_{6}\right)\right)_{2}\right) \\
& \sigma^{-1}\left(a_{3} S\left(a_{7}\right),\left(b_{3} S\left(b_{7}\right)\right)\left(a_{13} S\left(a_{15}\right)\right)\left(b_{12} S\left(b_{16}\right)\right)\right) \\
& \sigma^{-1}\left(\left(b_{4} S\left(b_{6}\right)\right)_{1},\left(a_{14} S\left(a_{16}\right)\right)\left(b_{13} S\left(b_{15}\right)\right)\right)\left(a_{5} b_{5} \otimes a_{15} b_{14}\right)
\end{aligned}
$$




$$
\begin{aligned}
& \left.=\sum \sigma^{-1}\left(a_{1} S\left(a_{5}\right), a_{6} S\left(a_{12}\right)\right) \sigma\left(a_{7} S\left(a_{11}\right), b_{1} S\left(b_{7}\right) b_{8} S\left(b_{12}\right)\right) \sigma\left(a_{2} S\left(a_{4}\right)\right), b_{3} S\left(b_{5}\right)\right) \\
& \sigma^{-1}\left(b_{2} S\left(b_{6}\right), a_{8} S\left(a_{10}\right) b_{9} S\left(b_{11}\right)\right)\left(a_{3} b_{4} \otimes a_{9} b_{10}\right) \\
& =\sum \sigma^{-1}\left(a_{1} S\left(a_{5}\right), a_{6} S\left(a_{12}\right)\right) \sigma^{-1}\left(\left(b_{1} S\left(b_{7}\right)\right)_{1},\left(b_{8} S\left(b_{12}\right)\right)_{1}\right) \sigma\left(\left(a_{7} S\left(a_{11}\right)\right)_{1},\right. \\
& \left.\left(b_{1} S\left(b_{7}\right)\right)_{2}\right) \sigma\left(\left(a_{7} S\left(a_{11}\right)\right)_{2}\left(b_{1} S\left(b_{7}\right)\right)_{3},\left(b_{8} S\left(b_{12}\right)\right)_{2}\right) \sigma\left(a_{2} S\left(a_{4}\right), b_{3} S\left(b_{5}\right)\right) \\
& \sigma^{-1}\left(\left(b_{2} S\left(b_{6}\right)\right)_{1}\left(a_{8} S\left(a_{10}\right)\right)_{1},\left(b_{9} S\left(b_{11}\right)\right)_{1}\right) \sigma^{-1}\left(\left(b_{2} S\left(b_{6}\right)\right)_{2},\left(a_{8} S\left(a_{10}\right)\right)_{2}\right) \\
& \sigma\left(\left(a_{8} S\left(a_{10}\right)\right)_{3},\left(b_{9} S\left(b_{11}\right)\right)_{2}\right)\left(a_{3} b_{4} \otimes a_{9} b_{10}\right) \\
& =\sum \sigma^{-1}\left(a_{1} S\left(a_{5}\right), a_{6} S\left(a_{18}\right)\right) \sigma^{-1}\left(\left(b_{1} S\left(b_{7}\right)\right)_{1},\left(b_{8} S\left(b_{12}\right)\right)_{1}\right) \sigma\left(a_{7} S\left(a_{17}\right),\left(b_{1} S\left(b_{7}\right)\right)_{2}\right) \\
& \sigma\left(\left(a_{8} S\left(a_{16}\right)\right)\left(b_{1} S\left(b_{7}\right)\right)_{3},\left(b_{8} S\left(b_{12}\right)\right)_{2}\right) \sigma\left(a_{2} S\left(a_{4}\right), b_{3} S\left(b_{5}\right)\right) \\
& \sigma^{-1}\left(\left(a_{9} S\left(a_{15}\right)\right)\left(b_{2} S\left(b_{6}\right)\right)_{1},\left(b_{9} S\left(b_{11}\right)\right)_{1}\right) \sigma^{-1}\left(\left(b_{2} S\left(b_{6}\right)\right)_{2}, a_{10} S\left(a_{14}\right)\right) \\
& \sigma\left(a_{11} S\left(a_{13}\right),\left(b_{9} S\left(b_{11}\right)\right)_{2}\right)\left(a_{3} b_{4} \otimes a_{12} b_{10}\right) \\
& =\sum \sigma^{-1}\left(a_{1} S\left(a_{5}\right), a_{6} S\left(a_{18}\right)\right) \sigma^{-1}\left(b_{1} S\left(b_{13}\right), b_{14} S\left(b_{22}\right)\right) \\
& \left(a_{7} S\left(a_{17}\right), b_{2} S\left(b_{12}\right)\right) \sigma\left(\left(a_{8} S\left(a_{16}\right)\right)\left(b_{3} S\left(b_{11}\right)\right), b_{15} S\left(b_{21}\right)\right) \sigma\left(a_{2} S\left(a_{4}\right), b_{6} S\left(b_{8}\right)\right) \\
& \sigma^{-1}\left(\left(a_{9} S\left(a_{15}\right)\right)\left(b_{4} S\left(b_{10}\right)\right), b_{16} S\left(b_{20}\right)\right) \sigma^{-1}\left(b_{5} S\left(b_{9}\right), a_{10} S\left(a_{14}\right)\right) \\
& \sigma\left(a_{11} S\left(a_{13}\right), b_{17} S\left(b_{19}\right)\right)\left(a_{3} b_{7} \otimes a_{12} b_{18}\right) \\
& =\sum \sigma^{-1}\left(a_{1} S\left(a_{5}\right), a_{6} S\left(a_{14}\right)\right) \sigma^{-1}\left(b_{1} S\left(b_{9}\right), b_{10} S\left(b_{14}\right)\right) \\
& \sigma\left(a_{7} S\left(a_{13}\right), b_{2} S\left(b_{8}\right)\right) \sigma\left(a_{2} S\left(a_{4}\right), b_{4} S\left(b_{6}\right)\right) \\
& \sigma^{-1}\left(b_{3} S\left(b_{7}\right), a_{8} S\left(a_{12}\right)\right) \sigma\left(a_{9} S\left(a_{11}\right), b_{11} S\left(b_{13}\right)\right)\left(a_{3} b_{5} \otimes a_{10} b_{12}\right) \\
& =\sum \sigma^{-1}\left(a_{1} S\left(a_{5}\right), a_{6} S\left(a_{12}\right)\right) \sigma^{-1}\left(b_{1} S\left(b_{9}\right),\left(b_{10} S\left(b_{12}\right)\right)\right. \\
& \sigma\left(a_{7} S\left(a_{11}\right), b_{2} S\left(b_{8}\right)\right) \sigma^{-1}\left(a_{2} S\left(a_{4}\right), b_{4} S\left(b_{6}\right)\right) \\
& \sigma^{-1}\left(b_{3} S\left(b_{7}\right), a_{8} S\left(a_{10}\right)\right)\left(a_{3} b_{5} \otimes a_{9} \cdot{ }_{\sigma} b_{11}\right) \\
& =\sum \sigma^{-1}\left(a_{1} S\left(a_{3}\right), a_{4} S\left(a_{10}\right)\right) \sigma^{-1}\left(b_{1} S\left(b_{7}\right), b_{8} S\left(b_{10}\right)\right) \\
& \sigma\left(a_{5} S\left(a_{9}\right), b_{2} S\left(b_{6}\right)\right) \sigma^{-1}\left(b_{3} S\left(b_{5}\right), a_{6} S\left(a_{8}\right)\right)\left(a_{2} \cdot{ }_{\sigma} b_{4} \otimes a_{7} \cdot{ }_{\sigma} b_{9}\right) \\
& =\sum \sigma^{-1}\left(a_{1} S\left(a_{3}\right), a_{4} S\left(a_{6}\right)\right) \sigma^{-1}\left(b_{1} S\left(b_{3}\right), b_{4} S\left(b_{6}\right)\right)\left[\left(a_{2} \otimes a_{5}\right) \cdot_{\sigma}\left(b_{2} \otimes b_{5}\right)\right] \\
& =\Delta_{\sigma}(a) \cdot T_{\sigma} \Delta_{\sigma}(b) \text {. }
\end{aligned}
$$

If $S^{2}=I$, then we have

$\cdot_{\sigma}(I \otimes S) \Delta_{\sigma}(a)$

$$
\begin{aligned}
& =\sum \sigma^{-1}\left(a_{1} S\left(a_{3}\right), a_{4} S\left(a_{6}\right)\right)\left(a_{2} \cdot \sigma\right. \\
& \left.=\sum\left(a_{5}\right)\right) \\
& =\varepsilon(a) .
\end{aligned}
$$


In a similar manner, we can show that

$$
{ }_{\sigma}(S \otimes I) \Delta_{\sigma}(a)=\varepsilon(a) .
$$

This completes the proof of Proposition 2.5.

Remark 2.6. Let $A$ be any Hopf algebra with trivial normal invertible 2-cocycle $\sigma=\varepsilon_{A \otimes A}$, then $A=A_{\sigma}$ as Hopf algebra and $T_{\sigma}$ becomes a classical twist.

We have the following important result.

COROllary 2.7. Let $A$ be a commutative Hopf algebra with a normal invertible 2-cocycle $\sigma$, then $A_{\sigma}$ is $T_{\sigma}$-Hopf algebra.

Proof. Invoking of $[8,1.5 .12]$, we see that $S^{2}=I$. It is obvious that the Corollary 2.7 is true.

\section{Some lemmas}

In [2], we can see that if $\sigma$ is a normal invertible 2-cocycle map on $A$, then $(A, \sigma)$ is not necessary a coquasitriangular Hopf algebra. In the same manner as [2], we can show that [2, Theorem 1.3] is true for a normal invertible 2-cocycle $\sigma$. List it following as a Lemma:

Lemma 3.1. Let $A$ be a Hopf algebra with a normal invertible 2-cocycle $\sigma$ on A. We set

$$
\lambda(a)=\sum \sigma\left(a_{1}, S\left(a_{2}\right)\right)
$$

for all $a \in A$. Then $\lambda$ is convolution invertible with

$$
\lambda^{-1}(a)=\sum \sigma^{-1}\left(S\left(a_{1}\right), a_{2}\right)
$$

Lemma 3.2. Let $A$ be a Hopf algebra with a normal invertible 2-cocycle $\sigma$, then we have

$$
\lambda^{-1}(a b)=\sum \lambda^{-1}\left(b_{2}\right) \lambda^{-1}\left(a_{2}\right) \sigma\left(a_{3}, b_{3}\right) \sigma\left(S\left(b_{1}\right), S\left(a_{1}\right)\right)
$$

for all $a, b \in A$.

Proof. We compute as following:

$$
\begin{aligned}
\lambda^{-1}(a b) & =\sum \sigma^{-1}\left(S\left(b_{1}\right) S\left(a_{1}\right), a_{2} b_{2}\right) \\
& =\sum \sigma^{-1}\left(S\left(b_{2}\right), S\left(a_{3}\right) a_{4} b_{3}\right) \sigma^{-1}\left(S\left(a_{2}\right), a_{5} b_{4}\right) \sigma\left(S\left(b_{1}\right), S\left(a_{1}\right)\right) \\
& =\sum \sigma^{-1}\left(S\left(b_{2}\right), b_{3}\right) \sigma^{-1}\left(S\left(a_{2}\right), a_{3} b_{4}\right) \sigma\left(S\left(b_{1}\right), S\left(a_{1}\right)\right)
\end{aligned}
$$




$$
\begin{aligned}
= & \sum \sigma^{-1}\left(S\left(b_{2}\right), b_{3}\right) \sigma^{-1}\left(S\left(a_{3}\right) a_{4}, b_{4}\right) \\
& \sigma^{-1}\left(S\left(a_{2}\right), a_{5}\right) \sigma\left(a_{6}, b_{5}\right) \sigma\left(S\left(b_{1}\right), S\left(a_{1}\right)\right) \\
= & \sum \sigma^{-1}\left(S\left(b_{2}\right), b_{3}\right) \sigma^{-1}\left(S\left(a_{2}\right), a_{3}\right) \sigma\left(a_{4}, b_{4}\right) \sigma\left(S\left(b_{1}\right), S\left(a_{1}\right)\right) \\
= & \sum \lambda^{-1}\left(b_{2}\right) \lambda^{-1}\left(a_{2}\right) \sigma\left(a_{3}, b_{3}\right) \sigma\left(S\left(b_{1}\right), S\left(a_{1}\right)\right) .
\end{aligned}
$$
have

Let $A$ be Hopf algebra, $\mathscr{M}$ denote the category of left $A$-comodule. We

Lemma 3.3 ([6, Theorem 4.1]). Let $(A, \sigma)$ be a coquasitriangular Hopf algebra. Then there is a braided group $\underline{A}$ in the category $\mathscr{M}$. As a coalgebra, $\underline{A}$ coincides with $A$. The algebra structure and the antipode are transmuted to

$$
\begin{aligned}
a \cdot b & =\sum \sigma\left(b_{1} S\left(b_{3}\right), S\left(a_{2}\right)\right) a_{1} b_{2}, \\
\underline{S}(b) & =\sum \sigma\left(S\left(b_{4}\right) S^{2}\left(b_{2}\right), b_{1}\right) S\left(b_{3}\right) .
\end{aligned}
$$

By [2], we can obtain that

LEMMA 3.4. Let $(A, \sigma)$ be a coquasitriangular Hopf algebra, then we have

$$
\begin{aligned}
a: b & =\sum \sigma\left(b_{3}, a_{2}\right) \sigma\left(b_{1}, S\left(a_{3}\right)\right) a_{1} b_{2}, \\
\underline{S}(b) & =\sum \sigma\left(S\left(b_{6}\right) b_{3}, b_{1}\right) \lambda^{-1}\left(b_{2}\right) \lambda\left(b_{4}\right) S\left(b_{5}\right) .
\end{aligned}
$$

Lemma 3.5. Let $A$ be a Hopf algebra with a normal invertible 2-cocycle $\sigma$. Then there is an isomorphism $\Psi: A^{\sigma} \rightarrow A_{\sigma}$ defined by

$$
\Psi(a)=\sum \sigma^{-1}\left(a_{1} S\left(a_{3}\right), a_{4}\right) a_{2}
$$

and $\Psi$ is an $A^{\sigma}$-comodule homomorphism, where all comodule structure maps are always coadjoint actions [9].

Proof. Firstly, we have

$$
\begin{aligned}
\Psi(a)= & \sum \sigma^{-1}\left(a_{1} S\left(a_{3}\right), a_{4}\right) a_{2} \\
& \sum \sigma^{-1}\left(a_{1}, S\left(a_{6}\right) a_{7}\right) \sigma^{-1}\left(S\left(a_{5}\right), a_{8}\right) \sigma\left(a_{2}, S\left(a_{4}\right)\right) a_{3} \\
= & \sum \sigma\left(a_{1}, S\left(a_{3}\right)\right) a_{2} \lambda^{-1}\left(a_{4}\right) .
\end{aligned}
$$

It is easy to show that $\Psi$ is invertible with

$$
\left.\Psi^{-1}(a)=\sum \sigma^{-1}\left(a_{1}\right), S\left(a_{4}\right)\right) a_{2} \lambda\left(a_{3}\right) .
$$

To check that $\Psi$ is an $A^{\sigma}$-comodule homomorphism, we calculate that 
$(I \otimes \Psi) \operatorname{Co}_{A^{\sigma}}(a)$

$$
\begin{aligned}
= & \sum a_{1} \cdot S^{\sigma}\left(a_{3}\right) \otimes \Psi\left(a_{2}\right) \\
= & \sum \sigma\left(a_{3}, S\left(a_{4}\right)\right) \sigma^{-1}\left(S\left(a_{6}\right), a_{7}\right)\left(a_{1} S\left(a_{5}\right) \otimes \Psi\left(a_{2}\right)\right) \\
= & \sum \sigma\left(a_{1}, S\left(a_{9}\right)\right) \sigma^{-1}\left(a_{3}, S\left(a_{7}\right)\right) \sigma\left(a_{5}, S\left(a_{6}\right)\right) \sigma^{-1}\left(S\left(a_{10}\right), a_{11}\right)\left(a_{2} S\left(a_{8}\right) \otimes \Psi\left(a_{4}\right)\right) \\
= & \sum \sigma\left(a_{1}, S\left(a_{8}\right)\right) \sigma^{-1}\left(a_{3}, S\left(a_{6}\right)\right) \lambda\left(a_{5}\right) \lambda^{-1}\left(a_{9}\right)\left(a_{2} S\left(a_{7}\right) \otimes \Psi\left(a_{4}\right)\right) \\
= & \sum \sigma\left(a_{1}, S\left(a_{11}\right)\right) \sigma^{-1}\left(a_{3}, S\left(a_{9}\right)\right) \lambda\left(a_{8}\right) \lambda^{-1}\left(a_{12}\right) \\
& \sigma\left(a_{4}, S\left(a_{6}\right)\right) \lambda^{-1}\left(a_{7}\right)\left(a_{2} S\left(a_{10}\right) \otimes a_{5}\right) \\
= & \sum \sigma\left(a_{1}, S\left(a_{9}\right)\right) \sigma^{-1}\left(a_{3}, S\left(a_{7}\right)\right) \sigma\left(a_{4}, S\left(a_{6}\right)\right) \lambda^{-1}\left(a_{10}\right)\left(a_{2} S\left(a_{8}\right) \otimes a_{5}\right) \\
= & \sum \sigma\left(a_{1}, S\left(a_{5}\right)\right) \lambda^{-1}\left(a_{6}\right)\left(a_{2} S\left(a_{6}\right) \otimes a_{3}\right) \\
= & C o_{A^{\sigma}} \Psi(a) .
\end{aligned}
$$

Lemma 3.6. Let $A$ be a Hopf algebra with a normal invertible 2-cocycle $\sigma$. Then $\Psi$ (same as in Lemma 3.5) is a coalgebra homomorphism.

Proof. We compute

$$
\begin{aligned}
\Delta_{\sigma} \Psi(a) & =\sum \sigma^{-1}\left(a_{1} S\left(a_{8}\right), a_{9}\right) \sigma^{-1}\left(a_{2} S\left(a_{4}\right), a_{5} S\left(a_{7}\right)\right)\left(a_{3} \otimes a_{6}\right) \\
& =\sum \sigma^{-1}\left(a_{1} S\left(a_{5}\right) a_{6} S\left(a_{10}\right), a_{11}\right) \sigma^{-1}\left(a_{2} S\left(a_{4}\right), a_{7} S\left(a_{9}\right)\right)\left(a_{3} \otimes a_{8}\right) \\
& =\sum \sigma^{-1}\left(a_{1} S\left(a_{3}\right), a_{4} S\left(a_{8}\right) a_{9}\right) \sigma^{-1}\left(a_{5} S\left(a_{7}\right), a_{10}\right)\left(a_{2} \otimes a_{6}\right) \\
& =\sum \sigma^{-1}\left(a_{1} S\left(a_{3}\right), a_{4}\right) \sigma^{-1}\left(a_{5} S\left(a_{7}\right), a_{8}\right)\left(a_{2} \otimes a_{6}\right) \\
& =(\Psi \otimes \Psi) \Delta(a) .
\end{aligned}
$$

\section{Braided group $\underline{A}^{\sigma}$}

Throughout this section, $A$ is always a commutative Hopf algebra with a normal invertible 2-cocycle $\sigma$. Lemma 2.2 says that $\left(A^{\sigma}, \tilde{\sigma}\right)$ is a coquasitriangular Hopf algebra. In this section we will show that the braided group $\underline{A}^{\sigma} \cong A_{\sigma}$.

Lemma 4.1. Let $A$ be a commutative Hopf algebra with a normal invertible 2-cocycle $\sigma$. Then $\Psi: \underline{A}^{\sigma} \rightarrow A_{\sigma}$ (defined as in Lemma 3.5) is an algebra homomorphism. 
Proof. For all $a, b \in A$, we have

$$
\begin{aligned}
& \Psi(a: b)=\sum \tilde{\sigma}\left(b_{1} \cdot S^{\sigma}\left(b_{3}\right), S^{\sigma}\left(a_{2}\right)\right) \Psi\left(a_{1} \cdot b_{2}\right) \\
& =\sum \tilde{\sigma}\left(b_{1} \cdot S^{\sigma}\left(b_{10}\right), S^{\sigma}\left(a_{9}\right)\right) \sigma\left(a_{1}, b_{2}\right) \sigma^{-1}\left(a_{8}, b_{9}\right) \sigma\left(a_{2} b_{3}, S\left(a_{4} b_{5}\right)\right) \\
& \lambda^{-1}\left(b_{7}\right) \lambda^{-1}\left(a_{6}\right) \sigma\left(a_{7}, b_{8}\right) \sigma\left(S\left(b_{6}\right), S\left(a_{5}\right)\right) a_{3} b_{4} \\
& =\sum \tilde{\sigma}\left(b_{1} \cdot S^{\sigma}\left(b_{11}\right), S^{\sigma}\left(a_{9}\right)\right) \sigma\left(a_{2} b_{3}, S\left(b_{7}\right)\right) \sigma\left(a_{3} b_{4} S\left(b_{6}\right), S\left(a_{5}\right)\right) \\
& \sigma\left(a_{7}, b_{9}\right) \sigma\left(a_{1}, b_{2}\right) \sigma^{-1}\left(a_{8}, b_{10}\right) \lambda^{-1}\left(a_{6}\right) \lambda^{-1}\left(b_{8}\right) a_{4} b_{5} \\
& =\sum \tilde{\sigma}\left(b_{1}, S^{\sigma}\left(a_{10}\right)\right) \tilde{\sigma}\left(S^{\sigma}\left(b_{11}\right), S^{\sigma}\left(a_{9}\right)\right) \sigma\left(a_{2} b_{3}, S\left(b_{7}\right)\right) \sigma\left(a_{3} b_{4} S\left(b_{6}\right), S\left(a_{5}\right)\right) \\
& \sigma\left(a_{7}, b_{9}\right) \sigma\left(a_{1}, b_{2}\right) \sigma^{-1}\left(a_{8}, b_{10}\right) \lambda^{-1}\left(a_{6}\right) \lambda^{-1}\left(b_{8}\right) a_{4} b_{5} \\
& =\sum \tilde{\sigma}\left(b_{1}, S^{\sigma}\left(a_{8}\right)\right) \tilde{\sigma}\left(b_{9}, a_{7}\right) \sigma\left(a_{2} b_{3}, S\left(b_{7}\right)\right) \sigma\left(a_{3} b_{4} S\left(b_{6}\right), S\left(a_{5}\right)\right) \\
& \sigma\left(a_{1}, b_{2}\right) \lambda^{-1}\left(a_{6}\right) \lambda^{-1}\left(b_{8}\right) a_{4} b_{5} \quad \text { (by definition of } S^{\sigma} \text { ) } \\
& =\sum \tilde{\sigma}\left(b_{1}, S\left(a_{10}\right)\right) \sigma\left(a_{8}, S\left(a_{9}\right)\right) \tilde{\sigma}\left(b_{9}, a_{7}\right) \sigma^{-1}\left(S\left(a_{11}\right), a_{12}\right) \sigma\left(a_{2} b_{3}, S\left(b_{7}\right)\right) \\
& \sigma\left(a_{3} b_{4} S\left(b_{6}\right), S\left(a_{5}\right)\right) \sigma\left(a_{1}, b_{2}\right) \lambda^{-1}\left(a_{6}\right) \lambda^{-1}\left(b_{8}\right) a_{4} b_{5} \quad \text { (by definition of } S^{\sigma} \text { ) } \\
& =\sum \tilde{\sigma}\left(b_{1}, S\left(a_{9}\right)\right) \tilde{\sigma}\left(b_{9}, a_{7}\right) \lambda\left(a_{8}\right) \lambda^{-1}\left(a_{10}\right) \sigma\left(a_{2} b_{3}, S\left(b_{7}\right)\right) \\
& \sigma\left(a_{3} b_{4} S\left(b_{6}\right), S\left(a_{5}\right)\right) \sigma\left(a_{1}, b_{2}\right) \lambda^{-1}\left(a_{6}\right) \lambda^{-1}\left(b_{8}\right) a_{4} b_{5} \\
& =\sum \tilde{\sigma}\left(b_{1}, S\left(a_{11}\right)\right) \tilde{\sigma}\left(b_{11}, a_{9}\right) \sigma^{-1}\left(a_{8}, b_{10}\right) \sigma\left(a_{7}, b_{9}\right) \lambda\left(a_{10}\right) \lambda^{-1}\left(a_{12}\right) \\
& \sigma\left(a_{2} b_{3}, S\left(b_{7}\right)\right) \sigma\left(a_{3} b_{4} S\left(b_{6}\right), S\left(a_{5}\right)\right) \sigma\left(a_{1}, b_{2}\right) \lambda^{-1}\left(a_{6}\right) \lambda^{-1}\left(b_{8}\right) a_{4} b_{5} \\
& =\sum \tilde{\sigma}\left(b_{1}, S\left(a_{6}\right)\right) \tilde{\sigma}\left(b_{5}, a_{4}\right) \sigma^{-1}\left(a_{3}, b_{4}\right) \\
& \lambda\left(a_{5}\right) \lambda^{-1}\left(a_{7}\right) \sigma\left(a_{1}, b_{2}\right) \Psi\left(a_{2} b_{3}\right) \quad \text { (by definition of } \Psi \text { ) } \\
& =\sum \sigma\left(S\left(a_{7}\right), b_{1}\right) \sigma^{-1}\left(b_{2}, S\left(a_{6}\right)\right) \tilde{\sigma}\left(b_{6}, a_{4}\right) \\
& \sigma^{-1}\left(a_{3}, b_{5}\right) \lambda\left(a_{5}\right) \lambda^{-1}\left(a_{8}\right) \sigma\left(a_{1}, b_{3}\right) \Psi\left(a_{2} b_{4}\right) \quad \text { (by definition of } \tilde{\sigma} \text { ) } \\
& =\sum \sigma\left(S\left(a_{8}\right), b_{1}\right) \sigma^{-1}\left(b_{2}, S\left(a_{7}\right)\right) \sigma\left(a_{4}, b_{6}\right) \sigma^{-1}\left(b_{7}, a_{5}\right) \\
& \sigma^{-1}\left(a_{3}, b_{5}\right) \lambda\left(a_{6}\right) \lambda^{-1}\left(a_{9}\right) \sigma\left(a_{1}, b_{3}\right) \Psi\left(a_{2} b_{4}\right) \quad \text { (by definition of } \tilde{\sigma} \text { ) } \\
& =\sum \sigma\left(S\left(a_{6}\right), b_{1}\right) \sigma^{-1}\left(b_{2}, S\left(a_{5}\right)\right) \sigma^{-1}\left(b_{5}, a_{3}\right) \lambda\left(a_{4}\right) \lambda^{-1}\left(a_{7}\right) \sigma\left(a_{1}, b_{3}\right) \Psi\left(a_{2} b_{4}\right) \\
& =\sum \sigma\left(S\left(a_{12}\right), b_{1}\right) \sigma^{-1}\left(b_{2}, S\left(a_{11}\right)\right) \sigma^{-1}\left(b_{10}, a_{9}\right) \sigma\left(a_{1}, b_{3}\right) \\
& \lambda\left(a_{10}\right) \lambda^{-1}\left(a_{13}\right) \sigma\left(b_{4} a_{2}, S\left(a_{6}\right)\right) \sigma\left(b_{5} a_{3} S\left(a_{5}\right), S\left(b_{7}\right)\right) \sigma\left(b_{9}, a_{8}\right) \\
& \lambda^{-1}\left(b_{8}\right) \lambda^{-1}\left(a_{7}\right) b_{6} a_{4} \quad \text { (by definition of } \Psi \text { and the commutativety) }
\end{aligned}
$$




$$
\begin{aligned}
& =\sum \sigma\left(S\left(a_{10}\right), b_{1}\right) \sigma^{-1}\left(b_{2}, S\left(a_{9}\right)\right) \sigma\left(a_{1}, b_{3}\right) \lambda\left(a_{8}\right) \lambda^{-1}\left(a_{11}\right) \\
& \sigma\left(b_{4} a_{2}, S\left(a_{6}\right)\right) \sigma\left(b_{5} a_{3} S\left(a_{5}\right), S\left(b_{7}\right)\right) \lambda^{-1}\left(b_{8}\right) \lambda^{-1}\left(a_{7}\right) b_{6} a_{4} \\
& =\sum \sigma\left(S\left(a_{8}\right), b_{1}\right) \sigma^{-1}\left(b_{2}, S\left(a_{7}\right)\right) \sigma\left(a_{1}, b_{3}\right) \lambda^{-1}\left(a_{9}\right) \\
& \sigma\left(b_{4} a_{2}, S\left(a_{6}\right)\right) \sigma\left(b_{5} a_{3} S\left(a_{5}\right), S\left(b_{7}\right)\right) \lambda^{-1}\left(b_{8}\right) b_{6} a_{4} \\
& =\sum \sigma\left(S\left(a_{8}\right), b_{1}\right) \sigma^{-1}\left(b_{2}, S\left(a_{7}\right)\right) \sigma\left(a_{1}, b_{4} S\left(a_{5}\right)\right) \lambda^{-1}\left(a_{9}\right) \sigma\left(b_{3}, S\left(a_{6}\right)\right) \\
& \sigma\left(b_{5} a_{2} S\left(a_{4}\right), S\left(b_{7}\right)\right) \lambda^{-1}\left(b_{8}\right) b_{6} a_{3} \quad \text { (by }\langle 1\rangle \text { and the commutativety) } \\
& =\sum \sigma\left(S\left(a_{6}\right), b_{1}\right) \sigma\left(a_{1}, b_{2} S\left(a_{5}\right)\right) \lambda^{-1}\left(a_{7}\right) \sigma\left(b_{3} a_{2} S\left(a_{4}\right), S\left(b_{5}\right)\right) \lambda^{-1}\left(b_{6}\right) b_{4} a_{3} \\
& =\sum \sigma\left(S\left(a_{6}\right), b_{1}\right) \sigma\left(a_{1}, S\left(a_{5}\right) b_{2}\right) \lambda^{-1}\left(a_{7}\right) \\
& \sigma\left(a_{2} S\left(a_{4}\right) b_{3}, S\left(b_{5}\right)\right) \lambda^{-1}\left(b_{6}\right) a_{3} b_{4} \\
& =\sum \sigma\left(a_{1}, S\left(a_{7}\right)\right) \sigma\left(a_{2} S\left(a_{6}\right), b_{1}\right) \lambda^{-1}\left(a_{8}\right) \\
& \sigma\left(a_{3} S\left(a_{5}\right) b_{2}, S\left(b_{4}\right)\right) \lambda^{-1}\left(b_{5}\right) a_{4} b_{3} \\
& =\sum \sigma\left(a_{1}, S\left(a_{5}\right)\right) \sigma\left(\left(a_{2} S\left(a_{4}\right)\right)_{1}, b_{1}\right) \lambda^{-1}\left(a_{6}\right) \\
& \sigma\left(\left(a_{2} S\left(a_{4}\right)\right)_{2} b_{2}, S\left(b_{4}\right)\right) \lambda^{-1}\left(b_{5}\right) a_{3} b_{3} \\
& =\sum \sigma\left(a_{1}, S\left(a_{5}\right)\right) \sigma\left(a_{2} S\left(a_{4}\right), b_{2} S\left(b_{4}\right)\right) \\
& \lambda^{-1}\left(a_{6}\right) \sigma\left(b_{1}, S\left(b_{5}\right)\right) \lambda^{-1}\left(b_{6}\right) a_{3} b_{3} \\
& =\sum \sigma\left(a_{1}, S\left(a_{3}\right)\right) \lambda^{-1}\left(a_{4}\right)\left(a_{2} \cdot{ }_{\sigma} b_{2}\right) \sigma\left(b_{1}, S\left(b_{3}\right)\right) \lambda^{-1}\left(b_{4}\right) \\
& =\Psi(a) \cdot{ }_{\sigma} \Psi(b) \text {. }
\end{aligned}
$$

Note that the braided group $\underline{A}$ has the structure of a $T$-Hopf algebra relative to the braiding $T$ in the category $\mathscr{M}$, where $T$ is defined by

$$
\begin{gathered}
T: A \otimes A \rightarrow A \otimes A ; \\
T(a \otimes b)=\sum\left(b_{2} \otimes a_{2}\right) \sigma\left(a_{1} S\left(a_{3}\right), b_{1} S\left(b_{3}\right)\right) .
\end{gathered}
$$

Therefore, we can show:

LemMa 4.2. Let $A$ be a commutative Hopf algebra with a normal invertible 2-cocycle $\sigma$. Then $\Psi: \underline{A}^{\sigma} \rightarrow A_{\sigma}$ (defined as in Lemma 3.5) preserves the twist map.

Proof. For all $a, b \in A$, we have

$(\Psi \otimes \Psi) T(a \otimes b)$

$$
=\sum(\Psi \otimes \Psi)\left(b_{2} \otimes a_{2}\right) \tilde{\sigma}\left(a_{1} \cdot S^{\sigma}\left(a_{3}\right), b_{1} \cdot S^{\sigma}\left(b_{3}\right)\right)
$$




$$
\begin{aligned}
= & \sum(\Psi \otimes \Psi)\left(b_{2} \otimes a_{2}\right) \tilde{\sigma}\left(a_{1} \cdot S\left(a_{5}\right), b_{1} \cdot S\left(b_{5}\right)\right) \\
& \left.\sigma\left(a_{3}, S\left(a_{4}\right)\right) \sigma^{-1}\left(S\left(a_{6}\right), a_{7}\right) \sigma\left(b_{3}, S\left(b_{4}\right)\right) \sigma^{-1}\left(S\left(b_{6}\right), b_{7}\right) \quad \text { (by definition of } S^{\sigma}\right) \\
= & \sum(\Psi \otimes \Psi)\left(b_{4} \otimes a_{4}\right) \tilde{\sigma}\left(a_{2} S\left(a_{8}\right), b_{2} S\left(b_{8}\right)\right) \\
& \sigma\left(a_{1}, S\left(a_{9}\right)\right) \sigma^{-1}\left(a_{3}, S\left(a_{7}\right)\right) \sigma\left(b_{1}, S\left(b_{9}\right)\right) \sigma^{-1}\left(b_{3}, S\left(b_{7}\right)\right) \\
& \left.\sigma\left(a_{5}, S\left(a_{6}\right)\right) \sigma^{-1}\left(S\left(a_{10}\right), a_{11}\right) \sigma\left(b_{5}, S\left(b_{6}\right)\right) \sigma^{-1}\left(S\left(b_{10}\right), b_{11}\right) \quad \text { (by definition of } \cdot\right) \\
= & \sum\left(b_{5} \otimes a_{5}\right) \sigma\left(b_{4}, S\left(b_{6}\right)\right) \lambda^{-1}\left(b_{7}\right) \sigma\left(a_{4}, S\left(a_{6}\right)\right) \lambda^{-1}\left(a_{7}\right) \\
& \tilde{\sigma}\left(a_{2} S\left(a_{11}\right), b_{2} S\left(b_{11}\right)\right) \sigma\left(a_{1}, S\left(a_{12}\right)\right) \sigma^{-1}\left(a_{3}, S\left(a_{10}\right)\right) \sigma\left(b_{1}, S\left(b_{12}\right)\right) \sigma^{-1}\left(b_{3}, S\left(b_{10}\right)\right) \\
& \sigma\left(a_{8}, S\left(a_{9}\right)\right) \sigma^{-1}\left(S\left(a_{13}\right), a_{14}\right) \sigma\left(b_{8}, S\left(b_{9}\right)\right) \sigma^{-1}\left(S\left(b_{13}\right), b_{14}\right)(\text { by definition of } \Psi) \\
= & \sum\left(b_{5} \otimes a_{5}\right) \sigma\left(b_{4}, S\left(b_{6}\right)\right) \sigma\left(a_{4}, S\left(a_{6}\right)\right) \tilde{\sigma}\left(a_{2} S\left(a_{8}\right), b_{2} S\left(b_{8}\right)\right) \\
& \sigma\left(a_{1}, S\left(a_{9}\right)\right) \sigma^{-1}\left(a_{3}, S\left(a_{7}\right)\right) \sigma\left(b_{1}, S\left(b_{9}\right)\right) \sigma^{-1}\left(b_{3}, S\left(b_{7}\right)\right) \\
& \sigma^{-1}\left(S\left(a_{10}\right), a_{11}\right) \sigma^{-1}\left(S\left(b_{10}\right), b_{11}\right) \\
= & \sum\left(b_{3} \otimes a_{3}\right) \tilde{\sigma}\left(a_{2} S\left(a_{4}\right), b_{2} S\left(b_{4}\right)\right) \sigma\left(a_{1}, S\left(a_{5}\right)\right) \sigma\left(b_{1}, S\left(b_{5}\right)\right) \lambda^{-1}\left(a_{6}\right) \lambda^{-1}\left(b_{6}\right) \\
= & \sum\left(b_{4} \otimes a_{4}\right) \sigma\left(a_{2} S\left(a_{6}\right), b_{2} S\left(b_{6}\right)\right) \sigma^{-1}\left(b_{3} S\left(b_{5}\right), a_{3} S\left(a_{5}\right)\right) \\
& \sigma\left(a_{1}, S\left(a_{7}\right)\right) \sigma\left(b_{1}, S\left(b_{7}\right)\right) \lambda^{-1}\left(a_{8}\right) \lambda^{-1}\left(b_{8}\right) \\
= & T_{\sigma}(\Psi \otimes \Psi)(a \otimes b)
\end{aligned}
$$

as required. This finishes our proof.

Note that both $\underline{A}^{\sigma}$ and $A_{\sigma}$ are Hopf algebra, by [7], the bialgebra map $\Psi$ between $\underline{A}^{\sigma}$ and $A_{\sigma}$ is automatically a Hopf algebra map. Therefore, we have established the main result in this paper.

THEOREM 4.3. Let $A$ be a commutative Hopf algebra with a normal invertible 2-cocycle $\sigma, A_{\sigma}$ be $T_{\sigma}$-Hopf algebra and $\underline{A}^{\sigma}$ be the braided group of $\left(A^{\sigma}, \tilde{\sigma}\right)$. Then $\underline{A}^{\sigma} \cong A_{\sigma}$ as $T$-Hopf algebras.

\section{REFERENCES}

[1] R. LARSON AND J. TowbER, Two dual classes of bialgebras related to the concepts of "quantum groups" and "quantum Lie algebras", Comm. Algebra, 19 (1991), 3295-3345.

[ 2] Y Dor, Braided bialgebras and quadratic bialgebras, Comm. Algebra, 21 (1993), 1731-1749.

[3] Y DoI AND M. TAKeUCHI, Multiplication alteration by two-cocycles (the quantum view), Comm. Algebra, 22 (1994), 5715-5732.

[4] S. MAJID, Quasitriangular Hopf algebras and Yang-Baxter equations, Internat. J. Modern Phys. A, 5 (1990), 1-91.

[ 5] D. E. RADFORD, Minimal quasitriangular Hopf algebra, J. Algebra, 157 (1993), 185-315. 
[6] S. MaJid, Braided groups, J. Pure Appl. Algebra, 86 (1993), 187-221.

[7] S. MAIID, Foundations of Quantum Group Theory, Cambridge University Press, Cambridge, 1995.

[ 8 ] S. Montgomery, Hopf Algebra and their Actions on Rings, CBMS Regional Conf. Ser. in Math., 82, American Mathematical Society, Providence, 1993.

[9] R. K. MolnaR, Semi-direct products of Hopf algebra, J. Algebra, 47 (1977), 29-51.

[10] M. E. SweEdLER, Hopf Algebras, Benjamin, New York, 1969.

DepartMENT OF MATHEMATics

HENAN NORMAL UNIVERSITY

XinXiang, Henan 453002

P.R. CHINA 